\title{
The Cepheid Galactic Internet
}

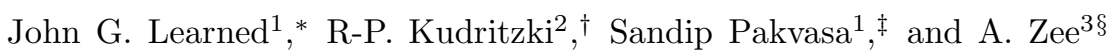 \\ ${ }^{1}$ Department of Physics and Astronomy, \\ University of Hawaii, 2505 Correa Road, \\ Honolulu, Hawaii 96822 U.S.A. \\ ${ }^{2}$ Institute for Astronomy, Astronomy, \\ University of Hawaii, 2680 Woodlawn Drive, \\ Honolulu, Hawaii 96822 U.S.A. \\ ${ }^{3}$ Kavli Institute for Theoretical Physics, \\ University of California, Santa Barbara, \\ California 93106 U.S.A.
}

(Dated: October 30, 2018)

\begin{abstract}
We propose that a sufficiently advanced civilization may employ Cepheid variable stars as beacons to transmit all-call information throughout the galaxy and beyond. One can construct many scenarios wherein it would be desirable for such a civilization of star ticklers to transmit data to anyone else within viewing range. The beauty of employing Cepheids is that these stars can be seen from afar (we monitor them out through the Virgo cluster), and any developing technological society would seem to be likely to closely observe them as distance markers. Records exist of Cepheids for well over one hundred years. We propose that these (and other regularly variable types of stars) be searched for signs of phase modulation (in the regime of short pulse duration) and patterns, which could be indicative of intentional signaling.

PACS numbers: 97.30.Gj,91.62.Fc,42.79.Sz,01.20.+x
\end{abstract}

\section{STAR TICKLERS AND THE NEED FOR A GALACTIC LIGHTHOUSE}

Considerations of communications with other life in the universe, the search for extraterrestrial intelligence (SETI) within (and beyond) our galaxy, have generally focused upon the use of microwaves and lasers as the communications medium. Problems arise from the difficult hurdles of resolution, noise and requisite power, making communications beyond our nearest neighbor stars very difficult, even with prior intention on both ends of a possible link. Searching for short (nanosecond) optical pulses shows some promise, though again suffers from the "needle-in-a-haystack" problem. Some authors [1] [2] [3] have proposed the use of neutrinos for avoiding some of the noise limitations, but large transmitter powers and formidable technical developments are still needed.

The problems with photons or neutrinos are not only data rate, but the ephemeral nature of the signal, if one is to contact an emergent civilization, one would have to

\footnotetext{
*Electronic address: jgl@phys.hawaii.edu

${ }^{\dagger}$ Electronic address: kud@ifa.hawaii.edu

${ }^{\ddagger}$ Electronic address: pakvasa@phys.hawaii.edu

$\S$ Electronic address: zee@kitp.ucsb.edu
}

waste enormous energy transmitting over aeons. A better scheme may be to leave artifacts for an emergent civilization to discover as it becomes capable. Recently, some authors have driven home the point that it is far more energetically practical for transmitting large amounts of data to place long lasting artifacts in stellar systems to which the ETI may wish communicate information (their history for example) as intelligent life matures and becomes capable of decoding this "Rosetta stone" [4].

That still leaves a role for timely communication: some "heads-up" type of communication informing newly technical societies of the existence of other life, perhaps of rules of engagement, instructions for finding said artifacts, or instructions on how to use some advanced means of communications (e.g. via extra-dimensions, or wormholes, or some other means beyond our present physics understanding). We cannot guess what the motivation might be, but given a number of credible possible motivations, let us speculate upon what sort of galactic lighthouse such a civilization might employ. We have speculated elsewhere [1] about the possibility of employing high energy neutrino beams directed specifically at a candidate stellar system, even a specific planet. This might be important if there are some security concerns by the transmitting ETI. Another possibility, if such concerns do not dominate, which we explore herein, is to construct some method to reach everyone in the galaxy 
(and beyond). In another context, the use of the cosmic microwave background to reach everyone in the universe was also considered [5] but as far as we know that is not within the capability of any inhabitants of the universe.

In the following, we propose that the well studied Cepheid variables might provide an easily and likely to be monitored transmitter, which would be seen by all societies undertaking serious astronomy.

\section{CEPHEID VARIABLES}

Cepheid variable stars was first observed in 1595. They were first recognized as having the marvelous property of having a relationship between period and luminosity by Henrietta Swan Leavitt [6] in 1908, permitting the establishment of a distance ladder on the galactic scale. The nearest stars could be ranged via parallax. Using the Cepheid scale one could move outwards up to stars in galaxies 20 megaparsec distant, and these stars have played a crucial role in the determination of the Hubble constant. Cepheids are generally bright stars with significant modulation and are easily observed. We expect that any civilization undertaking astronomy would soon discover them. Nor are there a daunting number of these, there being only of order 500 such stars presently tallied in our galaxy, and relatively few that are excellent standards.

The general picture for the Cepheids of Type I is that of a giant yellow star of population I with mass between five and ten times that of our sun, and $10^{3}$ to $10^{4}$ times the solar luminosity. A dozen or so of these stars are visible to the naked eye. The period of the brightness excursion ranges between 1 and 50 days, and is generally stable. The approximate period is given by the stellar dynamical time scale, which is just radius/velocity $=\left(R^{3} / G M\right)^{\frac{1}{2}}$. The period-luminosty relationship [7] is given by $M_{v}=-2.81 \log (P)-(1.43 \pm 0.1)$. The oscillation is explained by the build-up of ionized Helium, with increasing opacity with temperature, followed by violent expansion and de-ionization, and subsequent infall starting the cycle over again.

\section{HOW TO MODULATE CEPHEID PERIODS}

So, Cepheids form a class of readily detectable objects likely to be monitored by any emergent society which is undertaking astronomy. But can one modulate them?

The answer seems to us to be positive, because of the following. Any oscillator of this type, a blocking oscillator for example, depends upon some integration over time (as with a capacitor in an electrical analogy) followed by a non-linear breakdown or reset. The system undergoes a phase change, and of necessity in such systems there is a period of instability. A classic example of this is in heart beating [8], which can be triggered early with phase leading pulses. Near the time of normal triggering there is increased sensitivity to energy input.

We know, due to extensive modeling, that the solar luminosity is proportional to the $21^{\text {st }}$ power of the core temperature. In the sun the opacity decreases with temperature, and hence the sun is stable against perturbations. In Cepheids the opposite is true [9] 10] [11], so that small depositions of energy can lead to runaway conditions, exactly what occurs naturally as the star settles back down from the previous excursion. Delivery of a relatively small amount of energy at the right time can thus trip the cycle early. One would thus expect that an early pulse would have somewhat less amplitude than a normal cycle.

Neutrinos would seem to be the ideal delivery means for transporting a pulse of energy to the stellar core, both due to penetration ability and to speed. If one wanted to employ infalling material as the trigger, this disturbance would propagate at near the local speed of sound, and might well evaporate prior to reaching the core in any event. Neutrinos of about $1 \mathrm{TeV}$ would have an attenuation length of about $10^{6} \mathrm{~km}$ at solar densities. We have not modeled Cepheids in order to determine the optimum neutrino energy, but it is irrelevant for the present disucssion: we leave it as an engineering problem for the star tickling civilizations out there. The initial flavor mix of the neutrino beam makes little difference since oscillations will mix the flavors and in any event the cross-sections are flavor independent.

Most Cepheid studies have worked with some form of Fourier transform of the sampled optical brightness (stellar magnitude), often with observational gaps. For a regularly recurring pulsation with stable underlying period, this is adequate, and gaps can easily be dealt with. A long term periodogram will reveal the true period $(\tau)$. With phase advances present for encoding information, the long term Fourier transform will reveal a slightly shorter period on average, as determined by the magnitude and relative density of induced phase advances (call such period decrease $\tau \epsilon$ ). A simplest scheme would be with only one value of phase advance, a unique $\epsilon$, as opposed to a continuously variable or a set of integer values for $\epsilon$. For maximum information encoding, on average every other pulse would be shortened (a balanced set of ones and zeros). The information data rate $R$ would be then just one bit per period, reduced by half the average phase time shift: $R=1 /(\tau(1-\epsilon / 2))$. The data encoded in a given observational sample would then have a Fourier transform which would be spread about this mean $R$, with sidebands depending upon the sample window, data rate and such, but with a split peak. Given natural phase fluctuations, observational imprecision, and data windowing, the result may be hard to interpret. Indeed peculiar spectra are seen [12], some of which have surely to do with complicating companion stars and are not what we seek. Others have complex structures due to more complicated oscillation modes.

One standard method of Cepheid analysis involves 
Dworetsky's string minimizatio method [14]. Typically, a relatively small number of randomly spaced observations of a Cepheid are made over a long span of time. One guesses a trial period $\tau$ and plots the observed magnitude as a function of the phase as measured from the trial period. The points are then connected, giving a string in phase and magnitude space. The trial period is adjusted to achieve a minimum in this string length, thereby yielding both a period and a light-curve estimate. The points typically scatter about the mean envelope, and often points are discarded as outliers [12] [13] in order to find a smooth light-curve. Clearly such data manipulations could hide some unsuspected phase modulation of the data. In recent Cepheid studies some observers 15. determine the light curve by a process they call template fitting. This seems to be a good start for disentangling possible variations in the period which might be of a regular nature, but not discerned by Fourier transform.

We propose that a good way to examine this data for possible phase modulation would be to plot the histogram of measured pulse periods, deduced cycle by cycle, not averaged. This probably will result in some Gaussianlike distribution. If however the distribution is multiply peaked, in the most ideal case having two equal peaks with discernible separation, then we may suspect the possibility of intentional modulation. Should this be found, then we can interpret the identifications with one peak or the other as ones and zeros as in a binary encoded message. Thereafter the conclusive finding of an ETI signal would come about by identification of regularities which are hard to understand from any natural oscillator, such as involving repeated complex sequences, prime numbers, a limited "alphabet" (e.g. something akin to the familiar ASCII code), or even an apparent raster-like arrangement. At this point we cannot but fall back upon the supposition that we will recognize unnatural stellar fluctuations when we see them. In sum, we think the normal method of employing Fourier transforms (with windowing, gap filling, long term averaging, etc.) and other methods in common use to analyze the periodicity of Cepheids may cloud the discovery of intentional modulation, and that cycle-by-cycle periods need to be examined.

Of course with dense sampling of one or more observations per period, one can definitively disentangle the periods, pulse-by-pulse, and recover a signal should such be present. We have thought about the common situation of sparse observational sampling. We assume a simple saw toothed signal with longer and shorted periods, as illustrated in Figure 1 If one has a template for the waveform (light-curve, magnitude versus time), then with a single observation, one may extrapolate to (say) the descending zero crossing time, taking the mean magnitude as the zero point. These zero crossing times then will be integer multiples of the fundamental Cepheid period, if no modulation is taking place. If modulation is taking place, then the residuals will all be in clusters representing the number of cycles between observations.
On the other hand if there is single phase modulation the first pulse will be doubled, the second pulse cluster will be tripled and so on. This would clearly show evidence for binary cycle lengths. Of course more complicated multiple level modulation might be employed by the ETI. More difficult to discern would be the case of a continuous range of modulation. Note also that in the example cited above we can have evidence for possible modulation, without being necessarily able to decode a string of ones and zeros: the phase plot may tell us that the modulation is very peculiar.
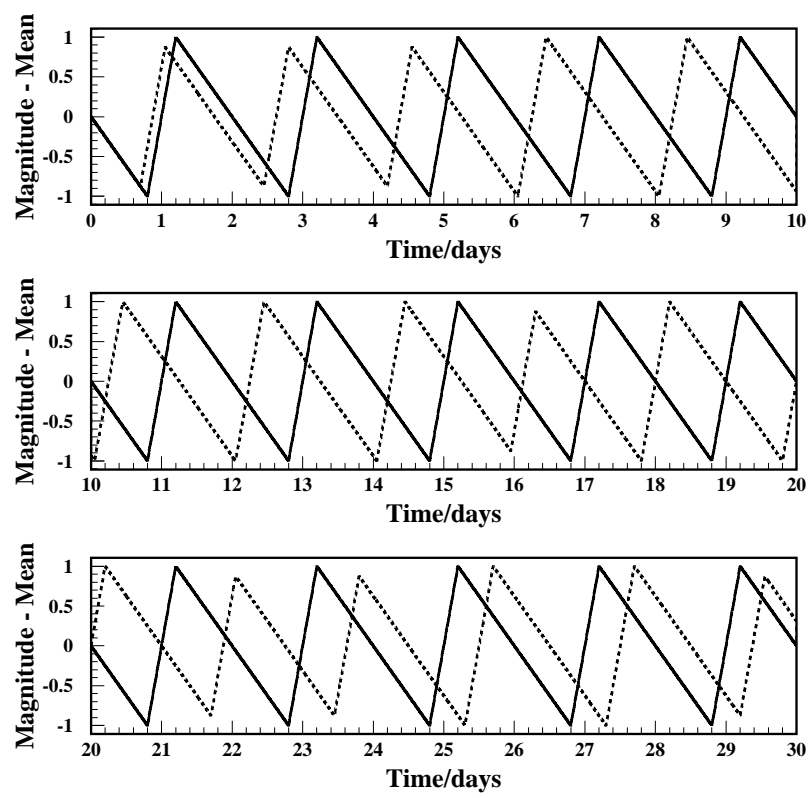

FIG. 1: Light curve of a simulated Cepheid vaiable. Ordinate is stellar magnitude relative to the mean, abscissa is time in days. The dashed curve represents an unmodulated (idealized) Cepheid with 2 day period and 2 magnitude luminosity excursion, with expansion taking 0.4 days. The solid curve represents an arbitrarily modulated light curve with triggered phase advance of 0.1 day ( 0.05 cycle). The units are, of course, arbitrary but representative of real data. The sharpness of the transitions which does not matter for the present discussions.

In Figure 2 we show the Fourier transforms (or more properly the periodogram) of the unmodulated case (a delta function as one would expect) and a modulated spectrum. In Figure 3 we illustrate the phase residual technique for both unmodulated and modulated stars. We take the period to be two days, with 1.8 days descending and 0.2 days expanding, and a total stellar magnitude range of 2.0. We take the modulation to be $5 \%$ in phase (or 0.1 days). The numbers are representative, but the actual choice has no particular meaning here, it simply illustrates the point that modulation will lead to peculiar phase residual plots.

This calculation is without question simplistic and 

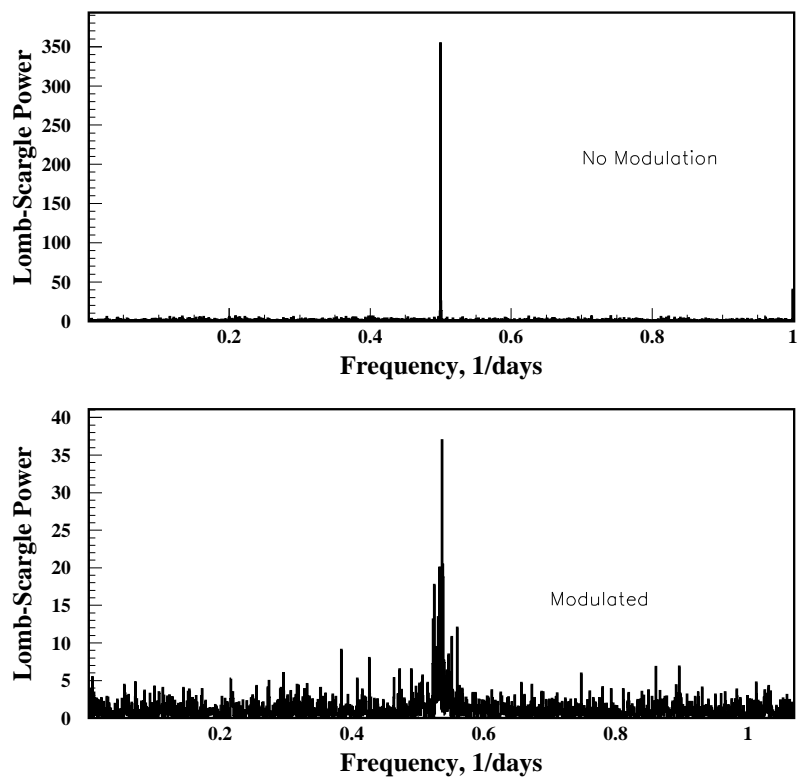

FIG. 2: Spectra of simulated observations of a regular periodic Cepheid variable and one with binary phase modulation. The ordinate is the Lomb-Scargle parameter, similar to chi squared; and abscissa is frequency. Note that the more complicated structure of the modulated case is not so obviously different from a noisey spectrum: one could not immediately discern that the latter case was not "natural".

meant to be merely illustrative. We imagine that experts in Cepheid analysis will be able to create more sophisticated algorithms. It may be noteworthy that there is a sort of inherent error correction coding in this type of modulation, in that the total phase offset with time tells one how many ones and zeroes have passed between observations.

\section{ENERGY COSTS}

Without detailed stellar modeling, we cannot make precise estimates of the amount of energy needed to trigger the Cepheid. A brute force upper limit would be the addition of some reasonable fraction of the core energy production over a time scale of the speed of sound traversing the core. Let us suppose that the pulse needs to be delivered in less than a second, and should be $10 \%$ or more of the stellar output. If we take the Cepheid period to be 100,000 seconds, then the average power input in neutrinos should be about $10^{-6}$ of the stellar output.

However, this is probably an overestimate, since one may deliver the neutrino beam to one side of the core, triggering the conversion locally, as with a spark plug. We do not know the gain achievable by this method, only that it is surely large: the Cepheid is a huge signal am-
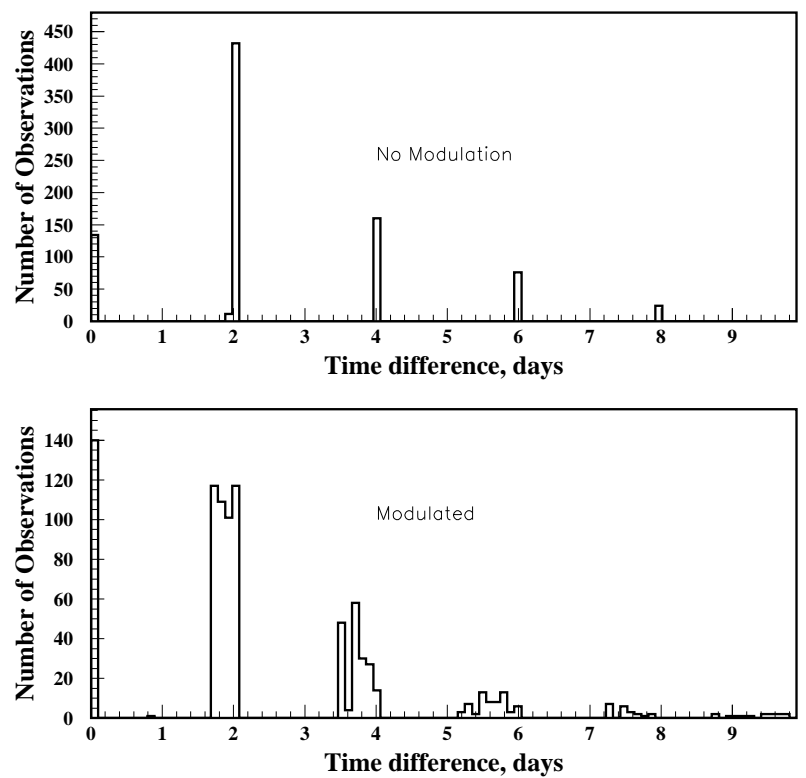

FIG. 3: Phase residuals of observations, when extrapolated to common phase at period given by Lomb-Scargle peak. The unmodulated data shows peaks for obervations in the next cycle, one skipped cycle, two missed cycles, etc. The modulated case shows splitting of these cases depending upon the combination of bits. This illustrates a possible means of detecting "unnatural" phase variation without dense sampling.

plifier.

There are also practical questions such as, how far away should the putative neutrino trigger generator be placed? It cannot be too close to the star or it will (if consisting of any technology with which we are familiar) melt. Such a device might sit out at a range of 100 AU, for example. Perhaps it consists of an advanced "solar energy" power station capable of utilizing the radiation of the Cepheid itself, accumulated over a full cycle and then dumped into the neutrino trigger pulse. Located at a distance of $100 \mathrm{AU}$ it would need to have receiving radius of about $0.2 \mathrm{AU}$. It is premature for us to speculate too much about this, being enough to recognize that such unstable systems present the opportunity to serve as gigantic signal amplifiers, and we presume that the advanced civilization will be able to accomplish this, and speculate that they find the prospect worthwhile.

\section{CONCLUDING REMARKS}

To summarize, in this note, we point out that that unstable stellar systems such as the Cepheids present an exciting opportunity to serve as gigantic signal amplifiers. We presume that a sufficiently advanced civilization will actually be able to tickle stars and we further speculate 
that they would find it worthwhile to use Cepheids as signaling devices. We propose that there may well be signatures of ETI communication available in data already recorded, and that a search of Cepheid (and perhaps other variable star, such as Lyrae) records may reveal an entre into the galactic internet!

It may be a long shot, but should it be correct, the payoff would be immeasurable for humanity. The beauty of this suggestion seems to be simply that the data already exists, and we need only look at the data in a new way.

\section{Acknowledgments}

We thank many colleagues for discussions of these ideas, in particular Freeman Dyson, Ralph BeckerSzendy, Walt Simmons, and Xerxes Tata. We would like to acknowledge support by the U.S.D.O.E. under grant DE-FG02-04ER41291 at the University of Hawaii, the N.S.F. under grant 04-56556 at the University of California at Santa Barbara.
[1] J.G. Learned, S. Pakvasa, and A. Zee, "Galactic Neutrino Communication", hep-ph arXiv:0805.2429. See also earlier literature cited therein.

[2] Z.K. Silagadze, "SETI and the muon collider", arXiv:0803.0409 (2008).

[3] J.G. Learned, S. Pakvasa, W. A. Simmons, and X. Tata, "Timing data communications with neutrinos: A new approach to SETI", Q.J.Roy.Astron.Soc. 35, 321 (1994).

[4] C. Rose and G. Wright, "Inscribed matter as an energy efficient means of communications with an extraterrestrial civilisation", Nature 41,431 (2004).

[5] S. Hsu and A. Zee, "Message in the Sky", Mod. Phys. Lett.A21, 1 (2006), arXiv:physics/0510102; "The NeverEnding Days of Being Dead" by Marcus Chown, Faber \& Faber (2007).

[6] H. S. Leavitt, "Ten Variable stars of the Algol type", Annals of Harvard College Observatory 60, 109 (1908). H. S. Leavitt and E. C. Pickering, " 28 New Variable Stars in the Harvard Map", Nos. 30 and 33; Harvard College Observatory Circular 142, 1(1908). G. Johnson, "Miss Leavitt's Stars", Norton (2005).

[7] M. W. Feast and R. M. Catchpole, "The Cepheid PL Zero-Point from Hipparcos Trigonometrical Parallaxes", Mon. Not. of the Roy. Astr. Soc., 286, Issue 1, L1 (1997).

[8] J. P. Wikswo, "Cardio Electrodynamics", (1999), http://online.kitp.ucsb.edu/online /colloq/wikswo1/oh/01.html
[9] S.A. Zhevakin, "Physical basis of the pulsation theory of variable star", Ann. Rev. Astro. Atrophys. 1963.1, 367-400 (1963).

[10] R.F. Christy, "Pulsation Theory", Ann. Rev. Astro. Astrophys. 1966.4: 353-392 (1966), and references therein for earlier ideas about pulsating stars.

[11] For a simple and readable account of the Eddington-Cox mechanism involving helium ionization and increased opacity, see R. A. Freedman and W. J. Kaufmann III, W. H. "Universe", 8th edition, Freeman \& Co., page 515 (2008).

[12] D.L. Welch and P.B. Stetson, "Robust variable star detection techniques suitable for automated searches: new results for NGC 1866", Ap. J. 105, 5, 1813 (1983).

[13] P.B. Stetson, "On the automatic Determination of LightCurve Parameters for Cepheid Variable", Pub. Astro. Soc. Pac. 108, 851 (1996).

[14] M.M. Dworetsky, "A period-finding method for sparse randomly spaced observations, or How long is a piece of string?", Mon. Not. R. Astr. Soc., 203, 917 (1983).

[15] N.R. Tanvir, M.A. Hendry, A. Watkins, S.M. Kanbur, L.N. Berdnikov and C.C. Ngeow, "Determination of Cepheid parameters by light-curve template-fitting", Mon. Not. R. Astron. Soc., arXiv:astro-ph/050404408v2 (2005). 